\title{
Health monitoring and wellness for all, a multichannel approach through innovative interfaces and systems
}

\author{
Giuliano Benelli, Giovanni Luca Daino, Alessandro Pozzebon, \\ Roberto Sesto and Riccardo Zambon \\ Department of Information Engineering \\ University of Siena \\ Siena, Italy \\ benelli, daino, alessandro.pozzebon, sesto, zambon@unisi.it
}

\begin{abstract}
In this paper, an innovative solution for remote monitoring of chronic disease is presented. This solution is based on experiences achieved in two research projects: the first one (named FIDES) founded by Tuscany Region in Italy, for promoting health monitoring decentralization for chronic diseases; and a second one (named T-Seniority) founded by European Commission focusing on ICT inclusion for elderly and disabled people. The solution presented in this paper is an open and modular platform, able to operate both at home and in structured environments (i.e. pharmacies, health centers, nursery houses, etc.). Concerning the home health monitoring issue, the adoption of TV as output interface draws huge benefits in terms of usability and familiarity, increasing the healthcare inclusion. Finally, this paper also introduces a new healthcare organizational model for chronic patient assistance through a comprehensive and capillary distributed healthcare monitoring system.
\end{abstract}

\section{INTRODUCTION}

In the second half of the nineteenth century, the growth of the economy has led to a significant increase in the quality of life of big portions of the world population, and subsequently to a dramatic growth of the life expectancy: looking at some statistics it is possible to see that, apart for some exceptions in some third world countries where the life expectancy at birth is lower than 60 years, in most part of the world this number is higher than 70, rising up to 75 and more in the western countries. A growth in the number of elderly people also means an increase in the number of people affected by chronic diseases, and then on the number of people who require continuous assistance. A possible help to reduce the costs and the timings of the assistance operations may come from the new ICT technologies appeared in the last years: the number of applications and services is becoming wide and differentiated, thanks mainly to the development of many digital devices with great communi- cation capabilities. On the other hand, the "digital gap" between people that are "info-skilled" and who have less access possibilities to those digital technologies such as elderly, disabled and other "info-marginalized" people, is constantly growing. In order to widen as much as possible the number of possible users of a home health monitoring system, here is introduced a new e-service provisioning model, adopting TV as the most familiar channel to reach "info marginalized" audiences. Specifically, the adoption a "hidden PC" into the living room scene as enhanced TV media center allows to introduce new interfaces and devices towards an easier and more familiar user interaction. Adopting this new solution, an innovative health monitoring system consisting of a platform provided with a set of devices measuring all the main vital parameters, to be positioned inside the pharmacies, is presented and tested. These parameters, once measured, became available both to the user, allowing him to check his health situation, and to the doctors, allowing the remote monitoring of a patient disease. This platform has been designed to be as much open as possible, in order to make easy the introduction of new monitoring devices. Furthermore, application layout and interfaces have been designed to allow the user to perform autonomously part of the measurements, in order to reduce as much as possible the impact of the system on the everyday life.

\section{THE FIDES PROJECT}

The FIDES project is a research program founded by Tuscany Region in Italy to develop a Telemedicine solution to be installed in pharmacies. The developed system allows the pharmacy customers to perform self-monitoring operations using a predefined set of medical analysis devices. The collected data are used to build what can be considered a sort of electronic case history of the user that is stored inside a centralized database collecting the information of all the people resident in Tuscany region. The FIDES project focused both on the realization of the monitoring platform and on the development of the data management inside what has been called the FIDES data bank. The FIDES monitoring platform, whose improvements and extensions are discussed in this paper, can roughly be subdivided in three parts: a Management System, an Authentication System and a Monitoring System. The Management System is the core of the platform: allows the user to interact with the platform, it collects data received from the sensors, it stores them inside a local database and transfers them to the central database. In the final prototype, an Asus eeeTop PC was used: this 
device integrates the same features of a common PC (Intel Atom D410 CPU, 1Gb RAM, 4 USB ports, 802.11n Wi-Fi interface, Bluetooth connection and Windows XP operating system) into a compact touch screen device (see Figure 1). The interface software has been developed using Java as a programming language: this solution allows the implementation of a flexible solution, easing all the network operations. Finally, the management system is connected to all the measurement devices: for this purpose different technologies have been studied. In the first prototype the wireless interfaces available are Bluetooth and ZigBee, while the wired ones are USB, serial RS232 and Ethernet. The Authentication system allows the identification of the user. This can be performed using three different technological solutions: the Bar code [1], the RFID [2] and the Smart Card [3]. The Monitoring System includes all the medical analysis devices used to measure the user's vital parameters. This is basically a Body Sensor Network composed by all the measurement devices, together with their interconnections with the Management System. While in the first prototype the set of sensors was fixed while the connections could be set up using different solutions, the enhancement described in this paper allows the realization of an open structure where the introduction of new devices is ready-made, while the interfaces are pre-defined in order to allow the standardization of the communication channels. The first prototype of the FIDES project allowed five different measurements: blood pressure, body weight, glycaemia, electrocardiogram and spirometry. While the measurement operations were carried out with commercial devices, for each device different communication solutions were tested. In particular, the following devices and the following communication technologies were tested:

- two blood pressure monitors by A\&D Company Ltd., a UA-767PC model with RS232 serial connection, and a UA-767PBC with Bluetooth connection. The first model was also modified and provided with a ZigBee $\mathrm{XBee}$ radio transmitter;

- two Health Scales by A\&D, an RS232 serial UC-321P model and a Bluetooth UC-321PBT model. The same modification as per the blood pressure monitors were made on the first model;

- a USB Glucocard G+ Meter by Menarini Diagnostic for the glycaemia. A ZigBee solution has been studied also for this device;

- a P8000 Electrocardiogram by Esaote, provided with an internal memory where up to 40 measurements can be stored, and with a serial RS232 connection;

- a USB Spiropalm spirometer by Cosmed.

\section{THE T-SENIORITY PROJECT}

The T-Seniority project provides a set of integrated care services through TV, oriented towards the elderly and/or disabled people. The same "User-friendly interfaces" are applied to all sorts of TV systems, taking into account the impairments in vision, hearing, mobility or dexterity. The model adopted introduces digital TV as an ICT services delivery channel, being it the most widely available, familiar

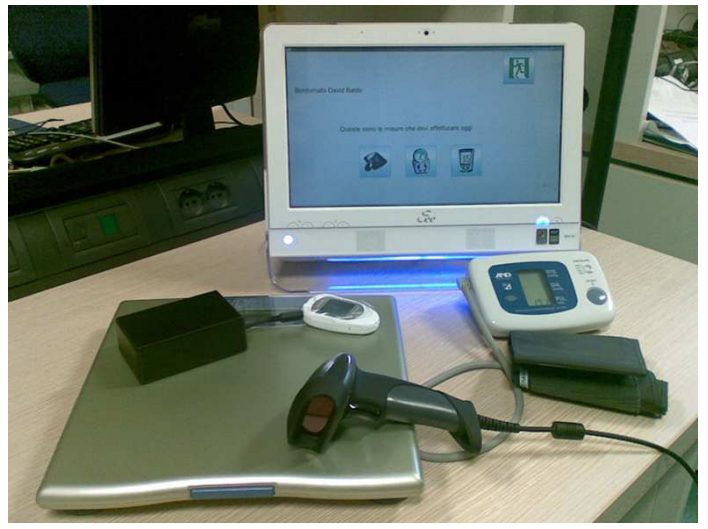

Figure 1: The FIDES platform with some measurement devices.

and preferred channel for info-marginalized audiences, so as to reach difficult-to-reach audiences, such as "disabled people getting older", who may have less access to other forms of digital technology (i.e. Internet, web applications and so on). The T-Seniority architecture is composed by a Central Server, representing the core of the application delivering all services, as shown in Figure 2. Furthermore, several Regional Servers are implemented, in order to manage the information exchange and the adaptation process for each national pilot implementation. These servers are interconnected with a Virtual Private Network (VPN). From the server side, T-Seniority services are delivered via the Internet. Concerning service delivery, the "on air" Digital TV infrastructure is needed for DTT (Digital Terrestrial TV) stream broadcasting for MHP (Multimedia Home Platform), IPHN (Internet Protocol Home Networking) and E-IPHN (Enhanced - IPHN) platforms. On the contrary, this feature is not required for IPTV (Internet Protocol TV) solution, where also the TV content is delivered through the Internet connection. In the MHP application provision, TSeniority services are available through an MHP application (xlet), broadcasted inside the DTT stream (carousel) over the air. Regarding personalized services, a return channel is adopted to connect the home device to the T-Seniority server (dial-up access for MHP, xDSL for IPTV/IPHN/E-IPHN).

The set of services available through the T-Seniority platform could be divided into public and personal services. Public services include general purpose services such as access to general interest information (i.e. weather, sport, news, etc.) and public care e-services repository about facilities, persons and resources (i.e. information on pharmacies timetable and locations, hospital facilities, health good practices, etc.). Personal services are available through user authentication. They include personal contents and customized information such as services for an independent living module (i.e. calls to relatives, messaging with care organizations, etc.), appointments (i.e. meeting with other people, market timetable, etc.), task lists (in order to schedule tasks during the day or the week) or shopping lists, reservations (i.e. medical analysis reservation, movie/theatre ticket reservation, etc.).

\section{CONVERGENCE}




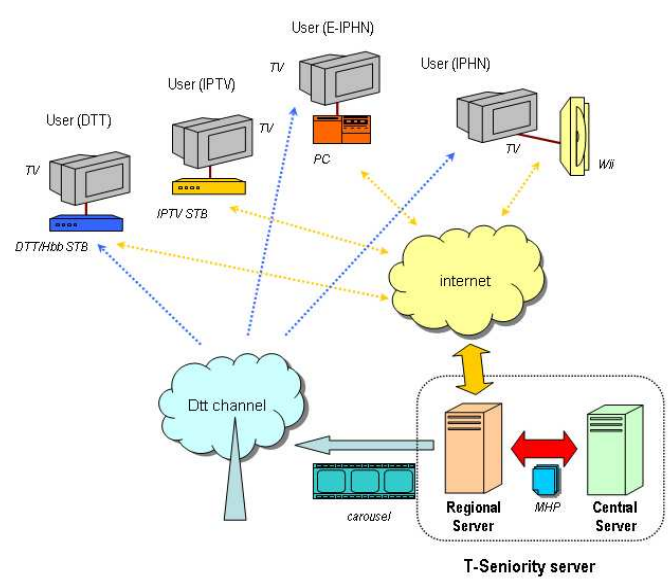

Figure 2: T-Seniority system architecture.

In this section the key innovation provided by this paper is described. The work focuses on the convergence between the FIDES project (by preserving the benefits introduced by this system for both patient monitoring and healthcare decentralization through health parameter measurement in pharmacies) and the T-Seniority project (by carrying on the usability benefits introduced by the adoption of digital TV for ICT service delivery to the elderly and disabled people). In the following, enhancements introduced to the solutions deriving from the two research project are presented and discussed. Finally, the comprehensive platform obtained by the convergence of the two solutions is presented.

\subsection{Enhancement from FIDES}

While the platform developed for the FIDES project represents a good starting point, some significant improvements have been carried out to enhance its flexibility and adaptability to different scenarios. A middleware has been introduced, in order to organize the introduction of new sensing devices. Two interfaces have been chosen, thorough which new devices can be easily connected with the Management System: wireless devices must connect with the system through a Bluetooth Channel, while wired devices are connected to the Management System through a USB hub. These two gates can be used by every kind of device: the data stream is automatically received by the Management System, that has then to decode it. The only additional work necessary to link a new sensing device to the system is then the interpretation module of the data stream, which is necessary to extract the information from the data packets obtained from the sensor. Once this setting has been stored inside the Management System, the data received from the new sensor can be added in the database storing the information about the patient. Once the system has been improved to be easily expanded, additional work has been carried out to turn it into a portable device. A hand-held version of the system has been studied and deployed, including all the devices stored inside a small case. In this case the Asus eeeTop PC has been replaced with a smaller Tablet PC. Then, a re-organization in the number and typology of sensors has been mandatory. Bigger sensing devices like the Scale and the Electrocardiogram have been removed, while the remaining devices have been positioned inside the case, in order to

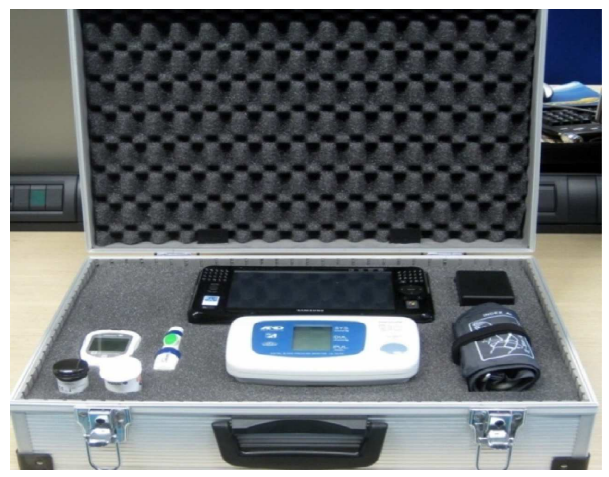

Figure 3: Hand-held FIDES platform.

reduce as much as possible the occupied space. Moreover, all the devices available with Bluetooth connections have been chosen, eliminating then most part of the wiring. The platform is shown in Figure 3 and has proven to be light and very portable, preserving anyway the performance levels of the fixed structure. Apart from the enhancements concerning the hardware and the interconnections, innovative features have been also introduced in the user interface. First of all, the system display interface has been designed to fit both big screens (i.e. such as TV, with big items and text for browsing from the sofa), and small screens. For this reason, the designed layout is suitable also for tablet screens, maintaining information readable and increasing both usability (i.e. through touchscreen intuitivity) and portability. Another key innovation is the introduction of the Video Conference capability, which allows the user to interact in real time with a healthcare operator. This feature notably increases the chances of autonomous use of the system. Besides, the platform is characterized by some other innovative concepts. Furthermore, FIDES benefits were extended also to Android devices, in order to exploit the market penetration of this platform and the wide range of available devices with different capabilities and prices. Another interesting solution is the adoption of Cisco Cius thin-client, an Android-based tablet which can be integrated in a communication station. Finally, voice recognition potentials could play a key role for enhancing usability in an easy way (i.e. considering Google voice recognition feature embedded in Android devices), especially in case of dexterity impairments.

\subsection{Enhancement from T-Seniority}

Here are reported the main improvements introduced in the T-Seniority E-IPHN solution in order to be integrated with the enhanced Health monitoring system described above.In this context the use of a full PC opens new scenarios in terms of capabilities, reaching an higher interactivity level beyond the common user interfaces. The core device is a full capabilities PC, maintaining the TV screen for displaying digital television contents and services as well. It can adopts different wired and wireless internet access networks (i.e. xDSL, Wi-Fi, WiMAX, HSPA). In this work, efforts are spent to run health measurement application and its components. In order to obtain a high usable experience, is needed to overtake the barriers of common hardware/software PC user interfaces (such as mouse, low-usability keyboard, visualization of contents on files/folders). Moreover, user inter- 


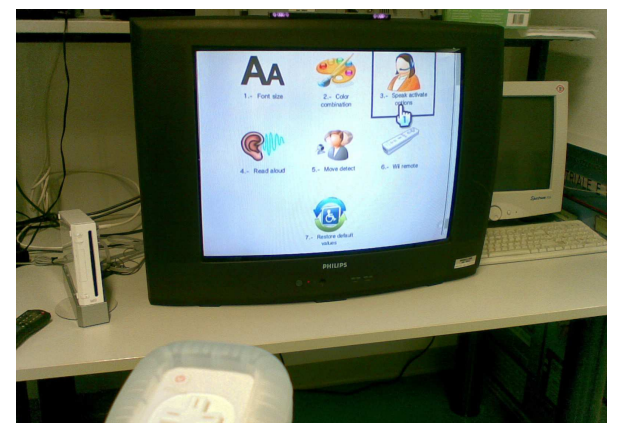

Figure 4: Health monitoring, wiimote navigation.

faces should be designed much simpler than usual PC, using functionalities for more intuitive interaction and information rendering. For these reasons, PC capabilities, information and interaction will be "filtered" by a more usable visualization mode, as a new layer between human and PC. Concerning input modalities, new devices could be introduced, such as wiimote-like devices (i.e. wiimote itself, eeestick or acer stick) or 3D mouses with a few buttons for an easier user interaction and more friendliness for elderly people (they can also be useful in case of mobility or dexterity impairments), an on screen QWERTY keyboard designed to be easily typed, associated with a word prediction algorithms for reducing typing at the minimum [4], or speech commands, useful for blind people. Regarding user identification, which is mandatory for accessing personal services, the unfriendly username-password insertion can be replaced by, biometrics identification (i.e. fingerprint, face, hand palm, iris, voice, $[5,6]$ ), specific movements [7], a personal USB key, Smartcards, RFID cards with pluggable readers or QR codes. As for common PCs, webcams allow video communication, and VoIP or videoconferencing are available through the platform. Concerning output interfaces, they should be suitably designed adopting several key features:

- Big icons and short sentences, easy to be understood and to be selected (with clear highlight of the selected item, for vision impairments);

- Tree menu, with a low number of icons (max 6 per level), or radial menu [8];

- The possibility of reading contents with short sentences of easy comprehension for blind people, and reading louder for hearing impairments;

- Inclusion of audio feedback (or vibrations), embedded in new controllers.

\section{CONCLUSIONS}

This paper describes the system realized through the convergence of a regional research project, which aims at promote the health monitoring decentralization for chronic diseases, and an European research project, which focuses on ICT inclusion for elderly and disabled people. The target of this work was the implementation of an open platform adopting a set of devices in order to measure all the main vital parameters. It allows self health monitoring, beyond the hospital to pharmacies, health centers and private homes. Furthermore, an innovative e-service provisioning model based on different Digital TV technologies is taken into account in order to provide health monitoring directly inside the patient home. Through this solution it is possible to joint the familiar TV device with PC-like capabilities, overcoming elderly's mistrust for new technologies and introducing them to ICT benefits, thus increasing their digital and social inclusion. In this work a step ahead in healthcare inclusion is moved: in a first phase, monitoring operations were moved from hospitals to pharmacies, starting healthcare decentralization. In a second phase the TV has introduced the ICT services (including health monitoring) in "info marginalized" people's homes, by adopting the "monitoring on the sofa" paradigm, thus providing capillary healthcare decentralization. In a third phase "mobile monitoring" is being introduced. Monitoring systems could be easily moved across rooms, across houses, they could be brought away for holidays, or carried on cars, etc., in order to finally enforce capillary healthcare decentralization. Through the described enhancement an innovative and comprehensive architecture for healthcare monitoring and care is realized and discussed. Laboratory testing on the platform have been carried out in order to evaluate input and output interfaces. The prototype as presented in this paper could be useful for domestic assistance as well for pharmacies, with a wide range of possible applicative fields.

\section{REFERENCES}

[1] R. C. Palmer, The Bar-code Book: Comprehensive guide to Reading, Printing, Specifying and Applying Bar Code and Other Machine-readable Symbols Helmers, Peterborough, 2001.

[2] K. Finkenzeller, RFID Handbook: Fundamentals and Applications in Contactless Smart Cards and Identification, JohnWiley and Sons, 2003.

[3] U. Hansmann, M. S. Nickolous, T. Schack, F. Selinger, Smart Card Application Development Using Java, Springer, 2000.

[4] Badr G., Raynal M., Optimized Interaction with Word Prediction List, in Proc. of the Association for the Advancement of Assistive Technology in Europe, Florence, Italy, p 871, 2009.

[5] M. Shirali-Shahreza, Login to internet websites by next generation game console, in Proc. of the IEEE/IFIP International Conference in Central Asia on Internet, Tashkent, Uzbekistan, pp 1-4, 2006

[6] M.S. Alam, and M. Akhteruzzaman, Real time fingerprint identification, in Proc. opf the IEEE National Aerospace and Electronics Conference, NAECON, Tuscaloosa, Alabama, USA, pp 433-440, 2000.

[7] M. Rafael Diaz, C. M. Travieso, J. B. Alonso and M. A. Ferrer, Biometric system based in the feature of hand palm, in Proc. of the International Carnahan Conference on Security Technology, Albuquerque, NM, USA, 2004.

[8] D. B. Dustin, R. Byers and J. J. LaViola, Evaluation of menu techniques using a $3 D$ game input device, in Proc. of the IEEE Symposium on 3D User Interfaces, 3DUI (2009). 